\title{
The Relationship Between Neuropathic Pain, and the Function of the Upper Limbs Based on Clinical Severity According to Electrophysiological Studies in Patients with Carpal Tunnel Syndrome
}

\author{
Motoki Sonohata $^{*}, 1$, Toshiyuki Tsuruta ${ }^{2}$, Hiroko Mine ${ }^{2}$, Tadatsugu Morimoto ${ }^{1}$ and \\ Masaaki Mawatari ${ }^{1}$ \\ ${ }^{1}$ Department of Orthopaedic Surgery, Faculty of Medicine, Saga University, 5-1-1 Nabeshima, Saga 849-8501, Japan \\ ${ }^{2}$ Tsuruta Orthopaedic Clinic, 1241-6 KatsuUshizu-Machi, Ogi City, Saga 849-0306, Japan
}

\begin{abstract}
Carpal tunnel syndrome (CTS) is an entrapment neuropathy of the median nerve, and CTS can cause neuropathic pain. The aim of this study was to evaluate the relationship between neuropathic pain, function of the upper limb, and the electrophysiology in patients with CTS. The terminal latency of median nerve was measured in 34 patients diagnosed with CTS, and they were asked to fill out the Japanese Society for Surgery of the Hand version of the Disability of Arm, Shoulder, and Hand questionnaire (DASH-JSSH) as the patient's assessment of the function of upper limbs and pain DETECT as an assessment for neuropathic pain. There was no significant correlation between the terminal latency and the pain DETECT score, or the terminal latency and the DASH-JSSH score. However, there was a significant correlation between the pain DETECT and DASH-JSSH scores. Neuropathic pain affects the function of the upper extremities in patients with CTS.
\end{abstract}

Keywords: Neuropathic pain, carpal tunnel syndrome, quality of life, painDETECT, electrophysiology, median nerve, terminal latency.

\section{INTRODUCTION}

Carpal tunnel syndrome (CTS) is an entrapment neuropathy of the median nerve at the wrist [1]. An assessment of patients with CTS has relied largely upon objective measurements including grip strength, muscle power, sensibility, Phalen's test, and Tinel's sign. Neurophysiological instruments for acquiring information on the conduction velocity of the median nerve are simple to use, and are also one of the most objective assessments and reproducible methods.

However, hand function is complex and these simple measurements may not reflect the patient's experience. The importance of the patient's assessment is well recognized [2]. A number of comprehensive assessment tools have been developed and validated $[3,4]$.

Neuropathic pain (NP) is a multifactor neurogenic disorder caused by physical damage to neurons, cancer and other diseases [5]. Furthermore, CTS is one of the causes of NP [6], and frequently manifests with NP [7]. NP is larger than a nociceptive pain, and may cause chronic pain [8].

The objective measurements, patient's assessment, and identification of NP are important to clearly articulate the pathophysiological status in patients with CTS.

*Address correspondence to this author at the Department of Orthopaedic Surgery, Faculty of Medicine, Saga University, Saga 849-8501, Japan; Tel: +81-952-34-2343; Fax: +81-952-34-2059;

E-mail: epc9719@yahoo.co.jp
The aim of this study was to determine the relationship between NP, function of the upper limb, and the electrophysiology in patients with CTS.

The study protocol adhered to the ethical guidelines of the 1975 Declaration of Helsinki, and the study was approved by the institutional review board of Tsuruta Orthopaedic Hospital.

\section{MATERIALS AND METHODOLOGY}

This study enrolled consecutive patients with newly diagnosed idiopathic CTS for more than 1 month between December 2011 and August 2012, and was conducted at a single center (Tsuruta Orthopaedic Clinic, Saga, Japan). Diagnosis was based on the characteristic symptoms and an electrophysiological study, and 63 hands in 61 patients were diagnosed with CTS. Two patients that had bilateral CTS were excluded. In addition 22 patients were excluded, because they had comorbid disease affecting the clinical evaluation of their upper limbs. One patient had the osteoarthritis of wrist joint on the affected side, one patient had a history of elbow fracture, and 21 patients had snapping fingers on their affected and/or opposite side from past to present. The remaining 36 patients were recruited for this study. Thirty-four patients that fully completed the questionnaire were enrolled in the study (Table 1). One patient received Pregabalin and 2 patients received nonsteroidal anti-inflammatory drugs which had been prescribed during a previous medical visit. In addition, 1 patient received Pregabalin and 12 patients received non-steroidal anti-inflammatory drugs from our institution. 
Table 1. Patient Characteristics

\begin{tabular}{|ll|c|}
\hline \multicolumn{2}{|l|}{ Parameter } & \multicolumn{2}{l|}{} \\
\hline \hline Sex (F/M) & mean (range) & $66(38-88)$ \\
\hline Age (years) & mean (range) & $27(1-180)$ \\
\hline Disease duration (months) & $17 / 17$ \\
\hline Affected side (right/left) & & $34 / 0$ \\
\hline Dominant hand (right/left) & & \\
\hline
\end{tabular}

An electrophysiological study measured the terminal latency of the median nerve using the Neuropack U (NIHON KOHDEN, Tokyo, Japan). The median nerve was stimulated at the halfway point between palmarislongus and flexor carpi radialis, and the derivation was measured on the abductor pollicisbrevis.

The patients were asked to fill out the Japanese Society for Surgery of the Hand version of the Disability of Arm, Shoulder, and Hand questionnaire (DASH-JSSH) [9] in order to assess the function of the upper limbs and pain DETECT [10] (PD) based on the patients' assessment of NP. The DASH is a suitable modality for measuring the health status outcome because it is mainly used as a measure of disability [11].

The DASH is a 30 -item scale that focuses at the patient' supper extremities [4]. Each item has five response choices, ranging from "no difficulty or no symptoms" to "unableto perform activity or very severe symptom." It is scored on a scale of 1-5. The items ask about the severity of each of the symptoms of pain, activity-related pain, tingling, weakness, and stiffness (five items: numbers 24-28); the degree of difficulty when performing various physical activities because of an arm, shoulder, or hand problem (21 items: numbers 1-21); the effect of the upper extremity problem on social activities, work, and sleep (three items: numbers 22 , 23, 29); and the psychological effect on their self-image (one item: number 30). These provide the DASH disability/symptom (DASHDS) score ranging from 0 (no disability) to 100 (the severest disability), after summation of the scores from all of the items and transformation.

The PD [10] was developed as a self-administered psychometric questionnaire to distinguish NP from non-NP among people with chronic low back pain. It is comprised of 7 items evaluating pain quality, one evaluating pain pattern, and one evaluating pain radiation, which all contribute to an aggregate score (range: -1 to 38 ). The PD has been validated against expert physician diagnosis of NP in people with a range of chronic pain conditions including 'typical' NP or 'typical' non-NP settings. The overall sensitivity, specificity, and positive predictive values using a cut-off score of 19 (NP symptoms $\geq 19$ ), are $85 \%, 80 \%$, and $83 \%$, respectively.

Additional questions used a visual analogue scale (VAS) to assess pain in 3 different ways; namely, the maximum VAS in the last 4 weeks, the average VAS in the last 4 weeks, and the current VAS.

All numerical data are expressed as the mean \pm standard deviation. All statistical analyses were conducted using the statistical package for social sciences (SPSS) version 19.0 for Windows (International Business Machines Corporation: IBM, NY, USA). Statistical significance was set at $\mathrm{p}<0.05$. Correlations were analyzed using Peason's product moment test.

\section{RESULTS}

The meanterminal latency was 9.2 \pm 3.8 (range 4.3-17.5) msec. The mean PD score was $9.4 \pm 7.8$ (range 0-28). The mean DASH-JSSH score was 64.0 25.3 (range 30-128). There was no significant correlation between the terminal latency and the PD score, or the terminal latency and the DASH-JSSH score (Figs. 1, 2). However, there is significant correlation between the PD and DASH-JSSH $(r=0.419$, $\mathrm{p}<0.05$ ) (Fig. 3).

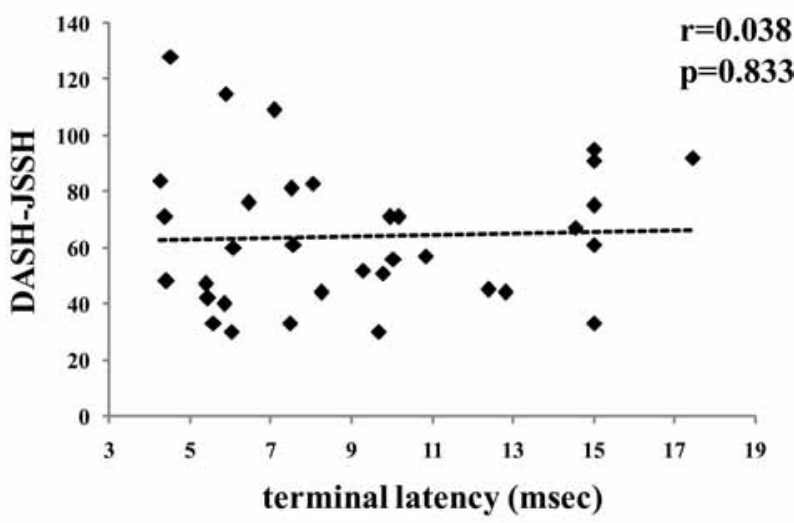

Fig. (1). Correlation between the terminal latency and the DASHJSSH score.

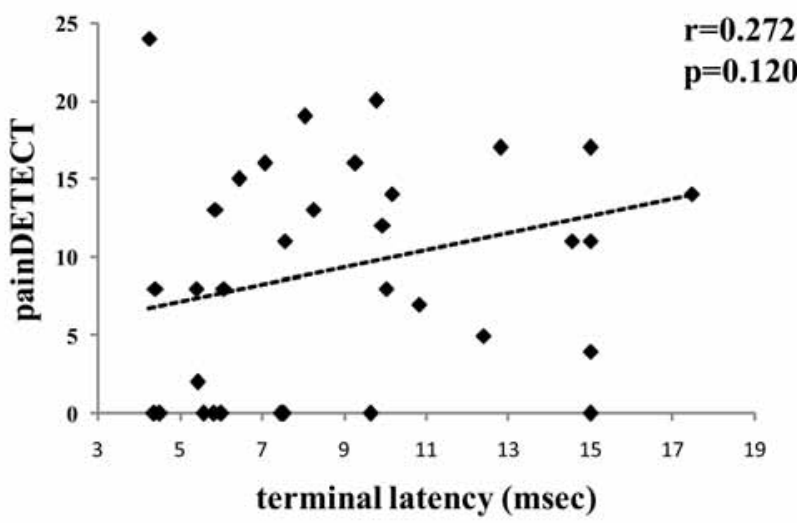

Fig. (2). Correlation between the terminal latency and the pain DETECT score.

The average of the maximum VAS in the last 4 weeks, the average VAS in the last 4 weeks, and the current VAS was43.9 \pm 36.9 (range 0-100 ) $\mathrm{mm}, 20.1 \pm 21.2$ (range 0-63) $\mathrm{mm}$, and 20.0 24.9 (range 0-78) $\mathrm{mm}$, respectively.

There was no significant correlation between the terminal latency and 3-way VAS. However, there was a significant correlation between the DASH-JSSH score and the average VAS in the last 4 weeks $(r=0.01)$. Furthermore, there was a significant correlation between the current DASH-JSSH and the 3-way VAS $(r=0.692, p<0.01 ; r=0.712, p<0.01 ; r=0.583$, $\mathrm{p}<0.01$, respectively; Table 2 ). 


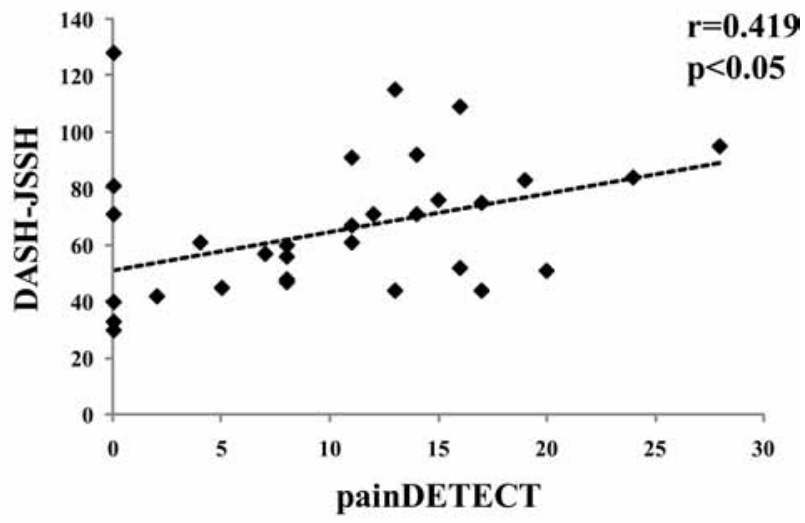

Fig. (3). Correlation between the painDETECT and DASH-JSSH scores.

Table 2. Correlation Between Visual Analogue Scale and Other Factors

\begin{tabular}{|c|c|c|c|}
\hline & TL $(\mathbf{m s})$ & DASH-JSSH & painDETECT \\
\hline \hline mean \pm SD & $9.1 \pm 3.8$ & $64 \pm 25$ & $9.4 \pm 7.8$ \\
range & $4.3-17.5$ & $30-128$ & $0-28$ \\
median & 8.0 & 60 & 8 \\
\hline VAS (1) & $\mathrm{r}=0.274$ & $\mathrm{r}=0.030$ & $\mathrm{r}=0.048$ \\
$43.9 \pm 37.0$ & $\mathrm{p}=0.206$ & $\mathrm{p}=0.892$ & $\mathrm{p}=0.828$ \\
$0-100$ & & & \\
47 & & & $\mathrm{r}=0.208$ \\
\hline VAS $(\mathbf{2})$ & $\mathrm{r}=0.313$ & $\mathrm{r}=0.164$ & $\mathrm{p}=0.340$ \\
$20.1 \pm 21.2$ & $\mathrm{p}=0.146$ & $\mathrm{p}=0.454$ & \\
$0-63$ & & & $\mathrm{r}=0.264$ \\
22 & & $\mathrm{r}=0.440$ & \\
\hline VAS (3) & $\mathrm{r}=0.295$ & $\mathrm{p}<0.05$ & \\
$20.0 \pm 24.9$ & $\mathrm{p}=0.172$ & & \\
$0-78$ & & & \\
6 & & & \\
\hline
\end{tabular}

Upper column: coefficient of correlation.

Lower column: $\mathrm{p}$ value.

TL: terminal latency.

VAS: Visual Analogue Scale.

DASH-JSSH: The Japanese Society for Surgery of the Hand version of the Disability of Arm, Shoulder, and Hand questionnaire.

VAS (1): maximum VAS in the last 4 weeks.

VAS (2): average VAS in the last 4 weeks.

VAS (3): current VAS.

\section{DISCUSSION}

This is a first report that investigated the relationship between NP, the function of the upper limb, and the electrophysiology in patients with CTS.

From 91 to $98 \%$ of the cases clinically diagnosed as CTS demonstrate abnormality on electrodiagnostic studies [11]. However, how the severity of electrophysiological study affects the clinical severity is unclear. The patient sometimes has no symptoms despite the presence of severe electrophysiological findings. On the contrary, severe symptoms may exist despite very mild electrophysiological findings. Some studies have described a significant relationship between the clinical severity and the severity of the electrophysiological findings in CTS, while others have found no significant correlation [13, 14]. Itsubo $\mathrm{T}$ et al. reported that both the electrophysiological responsiveness and DASH are highly responsive to treatment, however, they are not parallel [15].

Several instruments have been developed for the evaluation of upper extremity function, especially for patients with wrist and hand disorders [3, 4, 13, 16]. Some of them are disease-specific measures such as the Brigham and Women's Carpal Tunnel Questionnaire [13] or the Carpal Tunnel Syndrome Instrument [17, 18].

The most commonly used outcome measures for the wrist and hand region described in the literature and the best instrument for evaluating patients with disorders involving multiple joints of the upper limb is the DASH questionnaire [19]. Furthermore, the DASH is patient-rated and there is strong correlation with the 36-Item Short-Form Health Survey (SF-36) [9]. The current study found no significant correlation between the terminal latency of the median nerve and the DASH-JSSH. A conduction block of the median nerve did not directly affect the function of the upper limb.

There are screening tools for NP [20-22], and the PD is the one of the most useful assessment tool for NP [10]. The PD was developed and validated in adult low back pain, a condition with potentially mixed neuropathic and nociceptive pain mechanisms, and it does not include a physical examination component [10]. In addition, the PDis used for not only typical NP (i.e. spinal cord injury [23]), but also not typical NP (i.e. osteoarthritis [24]). Oncel et al. [12] found a correlation between the electrophysiology and the NP in the patients with CTS. They used the Washington NP Scale (NPS) for the scale of the NP, and the scale has 10 units; dull, cold, hot, itchy and so on. However, the relationship between NPS and PD, and the coefficient correlation was not very strong $(r=0.276, p<0.013)$. In addition, Rincon AI et al. reports there are no great differences in the quality of the pain among the cases with mild, moderate or severe CTS [25]. The current study found no significant correlation between the terminal latency of the median nerve and the PD. The severity of the electrophysiology did not directly affect the NP. We believe there are 2 clinically significant purposes to discriminate between nociceptive pain and NP in patients with CTS. One is that such discrimination is useful for determining the optimal medication (i.e. non-steroidal anti-inflammatory drugs, Pregabalin, or Tricyclic Antidepressants). While another purpose is to determine whether or not surgery is needed to avoid chronic pain with central sensitization due to protracted NP.

The number of enlisted patients was strictly reduced from 61 to 34 , thus, the limitation of this study was that the number of patients was too small to investigate the relationship between the NP and the patient's background. In addition, the terminal latency was used in the current study as a routine inspection in accordance with the standard regimen used at our institution, however, we should have compared sensory nerve conduction with the pain. Further studies with a larger cohort are therefore required. 


\section{CONCLUSION}

NP affects the function of upper extremities of patients with CTS. It is important to address the presence of NP in patients with CTS. In addition, the detection of NP is useful for selecting the optimal medication and surgical treatment.

\section{CONFLICT OF INTEREST}

The authors did not receive and will not receive any benefits or funding from any commercial party related directly or indirectly to the subject of this article.

\section{ACKNOWLEDGEMENTS}

The authors thank Ms. Masako Ikenaga and the hand therapist in TsurutaOrthopaedic Clinic for their contributions to this study.

\section{REFERENCES}

[1] Bland JD. Carpal tunnel syndrome. BMJ 2007; 335(7615): 343-6.

[2] Amadio PC, Rochester MN. Outcome research and the hand surgeon. J Hand Surg Am 1994; 19(3): 351-2.

[3] Hudak PL, Amadio PC, Bombardier C. Development of an upper extremity outcome measure: the DASH (disabilities of the arm, shoulder and hand) [corrected]. The Upper Extremity Collaborative Group (UECG). Am J Ind Med 1996; 29(6): 602-8.

[4] Chung KC, Pillsbury MS, Walters MR, Hayward RA. Reliability and validity testing of the Michigan hand outcomes questionnaire. $\mathbf{J}$ Hand Surg Am 1998; 23(4): 575-87.

[5] Jensen TS, Baron R, Haanpää $\mathrm{M}$, et al. A new definition of neuropathic pain. Pain 2011; 152(10): 2204-5.

[6] Scholz J, Woolf CJ. Can we conquer pain? Nat Neurosci 2002; 5(Suppl): 1062-7.

[7] Truini A, Padua L, Biasiotta A, et al. Differential involvement of A-delta and A-beta fibers in neuropathic pain related to carpal tunnel syndrome. Pain 2009; 145(1-2): 105-9.

[8] Caraceni A, Portenoy RK. An international survey of cancer pain characteristics and syndromes. IASP task force on cancer pain. International association for the study of pain. Pain 1999; 82(3): 263-74.

[9] Imaeda T, Toh S, Nakao Y, et al. Validation of the Japanese Society for Surgery of the HandVersion of the Disability of the Arm, Shoulder, and Hand Questionnaire. J Orthop Sci 2005; 10(4): 353-9.

[10] Freynhagen R, Baron R, Gockel U, Tolle T. pain DETECT: a new screening questionnaire to detect neuropathic components in patients with back pain. Curr Med Res Opin 2006; 22(10): 191120.
[11] Imaeda $\mathrm{T}$, Toh $\mathrm{S}$, Wada $\mathrm{T}$, et al. Impairment Evaluation Committee, Japanese Society for Surgery of the Hand. Validation of the Japanese Society for Surgery of the Hand Version of the Quick Disability of the Arm, Shoulder, and Hand (QuickDASHJSSH) questionnaire. J Orthop Sci 2006; 11(3): 248-53.

[12] Oncel C, Bir LS, Sanal E. The relationship between electrodiagnostic severity and Washington Neuropathic Pain Scale in patients with carpal tunnel syndrome. Agri 2009; 21(4): 146-8.

[13] Levine DW, Simmons BP, Koris MJ, et al. A self-administered questionnaire for the assessment of severity of symptoms and functional status in carpal tunnel syndrome. J Bone Joint Surg Am1993; 75(11): 1585-92.

[14] You H, Simmons Z, Freivalds A, Kothari MJ, Naidu SH. Relationships between clinical symptom severity scales and nerve conduction measures in carpal tunnel syndrome. Muscle Nerve 1999; 22(4): 497-501.

[15] Itsubo T, Uchiyama S, Momose T, Yasutomi T, Imaeda T, Kato H. Electrophysiological responsiveness and quality of life (QuickDASH, CTSI) evaluation of surgically treated carpal tunnel syndrome. J Orthop Sci 2009; 14(1): 17-23.

[16] MacDermid JC. Development of a scale for patient rating of wrist pain and disability. J Hand Ther 1996; 9(2): 178-83.

[17] Atroshi I, Johansson R, Sprinchorn A. Self-administered outcome instrument in carpal tunnel syndrome: reliability, validity and responsiveness evaluated in 102 patients. Acta Orthop Scand 1998; 69(1): 82-8.

[18] Imaeda T, Uchiyama S, Toh S, et al. Validation of the Japanese Society for Surgery of the Handversion of the carpal tunnel syndrome instrument. J Orthop Sci 2007; 12(1): 14-21.

[19] Changulani M, Okonkwo U, Keswani T, Kalairajah Y. Outcome evaluation measures for wrist and hand: which one to choose? Int Orthop 2008; 32(1): 1-6.

[20] Bennett MI, Smith BH, Torrance N, Potter J. The S-LANSS score for identifying pain of predominantly neuropathic origin: validation for use in clinical and postal research. J Pain 2005; 6(3): 149-58.

[21] Krause SJ, Backonja MM. Development of neuropathic pain questionnaire. Clin J Pain 2003; 19(5): 306-14.

[22] Bouhassira D, Attal N, Alchaar H, et al. Comparison of pain syndromes associated with nervous or somatic lesions and development of a new neuropathic pain diagnostic questionnaire (DN4). Pain 2005; 114(1-2): 29-36.

[23] Hallström H, Norrbrink C. Screening tools for neuropathic pain: can they be of use in individuals with spinal cord injury? Pain 2011; 152(4): 772-9.

[24] Hochman JR, Gagliese L, Davis AM, Hawker GA. Neuropathic pain symptoms in a community knee OA cohort. Osteoarthr Cartil 2011; 19(6): 647-54.

[25] de-la-Llave-Rincon AI, Laguarta-Val S, Arroyo-Morales M, Martinez-Perez A, Pareja JA, Fernandez-de-Las-Penas C. Characterisation of pain in patients with carpal tunnel syndrome according to electromyographic severity criteria. Rev Neurol 2012; 54(7): 407-14. 